\title{
Restframe UV-to-optical spectroscopy of APM 08279+5255
}

\section{BAL classification and black hole mass estimates *}

\author{
F. G. Saturni ${ }^{1,2}$, M. Bischetti ${ }^{1,3}$, E. Piconcelli ${ }^{1}$, A. Bongiorno ${ }^{1}$, C. Cicone ${ }^{4}$, C. Feruglio ${ }^{5}$, F. Fiore ${ }^{1}$,
} S. Gallerani ${ }^{6}$, M. Giustini ${ }^{7}$, S. Piranomonte ${ }^{1}$, G. Vietri ${ }^{1,8,9}$, and C. Vignali ${ }^{10,11}$

\author{
${ }^{1}$ INAF - Osservatorio Astronomico di Roma, Via Frascati 33, 00040 Monte Porzio Catone (RM), Italy \\ e-mail: francescogabriele.saturni@oa-roma.inaf.it \\ 2 Space Science Data Center, Agenzia Spaziale Italiana, Via del Politecnico snc, 00133 Roma, Italy \\ ${ }^{3}$ Dip. di Fisica, Università degli Studi di Roma "Tor Vergata”, Via della Ricerca Scientifica 1, 00133 Roma, Italy \\ ${ }^{4}$ INAF - Osservatorio Astronomico di Brera, Via Brera 28, 20121 Milano, Italy \\ ${ }^{5}$ INAF - Osservatorio Astronomico di Trieste, Via G. B. Tiepolo 11, 34143 Trieste, Italy \\ ${ }^{6}$ Scuola Normale Superiore, P.zza dei Cavalieri 7, 56126 Pisa, Italy \\ 7 SRON - Netherlands Institute for Space Research, Sorbonnelaan 2, 3584 CA Utrecht, The Netherlands \\ ${ }^{8}$ Excellence Cluster Universe, Technische Universität München, Boltzmannstr. 2, 85748 Garching b. München, Germany \\ ${ }^{9}$ European Southern Observatory, Karl-Schwarzschild-Str. 2, 85748 Garching b. München, Germany \\ ${ }_{11}^{10}$ Dip. di Fisica e Astronomia, Alma Mater Studiorum, Università degli Studi di Bologna, Via P. Gobetti 93/2, 40129 Bologna, Italy \\ 11 INAF - Osservatorio di Astrofisica e Scienza dello Spazio di Bologna, Via P. Gobetti 93/3. 40129 Bologna, Italy
}

Received 8 February 2018 / Accepted 24 April 2018

\begin{abstract}
We present the analysis of the restframe optical-to-UV spectrum of APM 08279+5255, a well-known lensed broad absorption line (BAL) quasar at $z=3.911$. The spectroscopic data were taken with the optical DOLoRes and near-IR NICS instruments at TNG, and include the previously unexplored range between $\mathrm{C}$ III] $\lambda 1910$ and [O III] $\lambda \lambda 4959,5007$. We have investigated the possible presence of multiple BALs by computing "balnicity" and absorption indexes (i.e., BI, $\mathrm{BI}_{0}$, and AI) for the transitions Si IV $\lambda 1400, \mathrm{C}$ IV $\lambda 1549, \mathrm{Al}$ III $\lambda 1860$, and $\mathrm{Mg}$ II $\lambda 2800$. No clear evidence for the presence of absorption features is found in addition to the already known, prominent BAL associated to C IV, which supports a high-ionization BAL classification for APM $08279+5255$. We also studied the properties of the [O III], $\mathrm{H} \beta$, and $\mathrm{Mg}$ II emission lines. We find that $[\mathrm{O} \mathrm{III}]$ is intrinsically weak $\left(F_{[\mathrm{OIII}]} / F_{\mathrm{H} \beta} \lesssim 0.04\right)$, as it is typically found in luminous quasars with a strongly blueshifted C IV emission line $\left(\sim 2500 \mathrm{~km} \mathrm{~s}^{-1}\right.$ for APM $\left.08279+5255\right)$. We computed the single-epoch black hole mass based on $\mathrm{Mg}$ II and $\mathrm{H} \beta$ broad emission lines, finding $M_{\mathrm{BH}}=(2 \div 3) \times 10^{10} \mu^{-1} M_{\odot}$, with the magnification factor $\mu$ that can vary between 4 and 100 according to $\mathrm{CO}$ and restframe UV-to-mid-IR imaging respectively. Using a Mg II equivalent width (EW)to-Eddington ratio relation, the $\mathrm{EW}_{\mathrm{MgII}} \sim 27 \AA$ measured for APM $08279+5255$ translates into an Eddington ratio of $\sim 0.4$, which is more consistent with $\mu=4$. This magnification factor also provides a value of $M_{\mathrm{BH}}$ that is consistent with recent reverberation-mapping measurements derived from C IV and Si IV.
\end{abstract}

Key words. galaxies: active - quasars: general - quasars: absorption lines - quasars: emission lines quasars: supermassive black holes - quasars: individual: APM 08279+5255

\section{Introduction}

APM 08279+5255 is a well-known luminous broad absorptionline quasar (BAL QSO) at $z=3.911$. Serendipitously discovered in a Galactic survey for cold carbon stars (Irwin et al. 1998), it is archetypal to several categories of the quasar class, showing together many of the observational phenomena that can be found in such objects. In fact, beyond having evidence of both broad (Srianand \& Petitjean 2000) and intrinsic narrow (Ellison et al. 2004) absorption features associated with the C IV $\lambda 1549$ emission line, it also shows an uncommon O vI $\lambda 1030$ BAL embedded in the Ly $\alpha$ forest (Hines et al. 1999) and an X-ray ultra-fast outflow (UFO) associated to highly-ionized iron (Hasinger et al. 2002; Chartas et al. 2002; Saez et al. 2009; Hagino et al. 2017). Furthermore, its high-ionization emission lines, from $\mathrm{Ly} \alpha$ to $\mathrm{C}$ III] $\lambda 1910$, are characterized by a significant

\footnotetext{
* The reduced spectrum is only available at the CDS via anonymous ftp to cdsarc.u-strasbg.fr (130.79.128.5) or via http://cdsarc.u-strasbg.fr/viz-bin/qcat?J/A+A/617/A118
}

blueshift of $\sim 2500 \mathrm{~km} \mathrm{~s}^{-1}$ with respect to molecular (Downes et al. 1999) and Balmer lines (Oyabu et al. 2009). A blueshifted emission component with $v \sim-800 \mathrm{~km} \mathrm{~s}^{-1}$, corresponding to a molecular outflow, is also detected in the $\mathrm{CO}(4-3)$ transition by Feruglio et al. (2017) through $3.2 \mathrm{~mm}$ observations with the NOEMA interferometer.

The source APM $08279+5255$ is gravitationally lensed (Ledoux et al. 1998) by an unobserved galaxy at $z \sim 1$ (Petitjean et al. 2000; Ellison et al. 2004). The lensed image is elongated in the NE direction and consists of three components, with a maximum separation of $0^{\prime \prime} .35 \pm 0^{\prime \prime} .02$ (Ledoux et al. 1998). This makes APM 08279+5255 the first confirmed case with an odd number of images (Ibata et al. 1999; Lewis et al. 2002b). The lack of knowledge about the lensing object further complicates the estimation of the magnification factor $\mu$. Current lens models are built on the observation of the $\mathrm{CO}(1-0)$ molecular line (e.g., Lewis et al. 2002a), but the resulting $\mu$ is strongly dependent on the lens geometry, ranging from $\mu \sim 4$ (highlyinclined spiral galaxy; Riechers et al. 2009) up to $\mu \sim 100$ 
(naked cusp; Egami et al. 2000). Nevertheless, with an intrinsic bolometric luminosity in the range of $5 \times 10^{13}$ to $10^{15} L_{\odot}$ APM $08279+5255$ can be considered among the most intrinsically luminous quasars.

Since its discovery, several photometric and spectroscopic observational campaigns have targeted APM 08279+5255 in different energy bands. Both short-term and long-term monitoring of this object have been performed for a wide range of purposes, from the analysis of its optical variability (Lewis et al. 1999) to the study of the UFO through photoionization codes (Saez \& Chartas 2011), the investigation of the C IV absorption variability (Trevese et al. 2013; Saturni et al. 2014) and the reverberation mapping (Trevese et al. 2007; Saturni et al. 2016). In particular, the variability study of APM $08279+5255$ absorption systems (Trevese et al. 2013; Saturni et al. 2014, 2016) concluded that the $\mathrm{C}$ IV absorption variability in APM $08279+5255$ is most likely driven by changes in the photoionization state of the gas, responding to variations of the $\mathrm{C}$ IV ionizing continuum level.

Single-epoch observations of APM $08279+5255$ include restframe UV high-resolution spectroscopy with Keck/HIRES (Ellison et al. 1999) and HST/STIS (Lewis et al. 2002b) for the study of the damped Ly $\alpha$ absorbers (DLAs) and intervening absorption systems (Petitjean et al. 2000; Ellison et al. 2004). Srianand \& Petitjean (2000) first analyzed the high-velocity absorption system blueward the $\mathrm{C}$ IV emission peak thanks to the availability of the Keck/HIRES spectrum, finding narrow absorption lines embedded between two unresolved broad components. Using the same spectrum, Ellison et al. (2004) studied the resolved absorption feature on the $\mathrm{C}$ IV red wing, classifying it as a system of four intervening clouds located close to the quasar systemic redshift.

In this paper, we present the quasi-simultaneous restframe optical-to-ultraviolet (UV) spectrum of APM 08279+5255 taken at the $3.5 \mathrm{~m}$ Telescopio Nazionale Galileo (TNG) in La Palma (Canarian Islands) with the Device Optimized for Low Resolution (DOLoRes; $\lambda / \Delta \lambda \sim 700$ ) and the Near-Infrared Spectrograph and Camera (NICS; $\lambda / \Delta \lambda \sim 500$ ). Covering the region between $\mathrm{C}$ III] and [O III] $\lambda \lambda 4959,5007$ which was unobserved so far, this broadband spectrum allows the study of the restframe wavelength range $\lambda \lambda \sim 1000-5000 \AA$ in a single state of quasar activity. In fact, the interval of 76 days between the near-infrared (NIR) and optical observations corresponds to a restframe interval of $\sim 15$ days, much shorter than typical variability timescales of APM 08279+5255 ( $\sim 30$ restframe days for continuum flux changes; e.g., Saturni et al. 2016). The paper is organized as follows: we describe the observations and the procedure of data reduction in Sect. 2; we analyze the spectral features in Sect. 3; finally, in Sect. 4 we present single-epoch mass estimates of the supermassive black hole (SMBH) hosted in APM 08279+5255, and discuss our results in Sect. 5. Throughout the text, we report all errors at $1 \sigma$ confidence level, and adopt a concordance cosmology with $H_{0}=70 \mathrm{~km} \mathrm{~s}^{-1} \mathrm{Mpc}^{-1}, \Omega_{\mathrm{M}}=0.3$ and $\Omega_{\Lambda}=0.7$.

\section{Observations and data reduction}

Observations of the APM 08279+5255 restframe optical-to-UV spectrum were carried out on 2011 February 19-20 (optical) and 2011 May 05 (UV) at TNG. The restframe UV spectrum of APM $08279+5255$ was obtained with the $R$-band grism (wavelength range $\lambda \lambda 4470-10073 \AA$, dispersion of $2.61 \AA \mathrm{px}^{-1}, \lambda / \Delta \lambda=714$ ) of the DOLoRes instrument, coupled to the $1^{\prime \prime}$ slit. The restframe optical spectrum was acquired with the NICS instrument in two low-resolution configurations, respectively for the $I J$ (wavelength range $\lambda \lambda 9000-14500 \AA$, dispersion of $5.5 \AA \mathrm{px}^{-1}$, $\lambda / \Delta \lambda=500$ ) and $H K$ (wavelength range $\lambda \lambda 14000-25000 \AA$, dispersion of $11.2 \AA \mathrm{px}^{-1}, \lambda / \Delta \lambda=500$ ) bands, with the same slit width of the $R$-band spectrum.

The restframe UV spectrum $\lambda \lambda 1020-1870 \AA$ considered in our analysis extends over the observed wavelength interval $\lambda \lambda \sim 5000-9200 \AA$. A large contamination due to overlap of higher spectral orders is visible redward $\lambda \sim 9200 \AA$. The spectrum was calibrated with standard IRAF procedures, and was cleaned from the major telluric absorptions, namely the Fraunhofer $\mathrm{A}$ and $\mathrm{B}$ bands and the $\mathrm{H}_{2} \mathrm{O}$ features, adopting the method described in Trevese et al. (2013). The total restframe optical spectrum extends over the observed wavelength intervals $\lambda \lambda \sim 8700-14500 \AA$ ( $I J$ bands) and $\lambda \lambda \sim 13500-24700 \AA(H K$ bands), corresponding to $\lambda \lambda 17705030 \AA$ in the restframe UV-tooptical bands. A standard NICS observing sequence consists of exposures at two different dither positions $(A$ and $B)$ and taken in the pattern $A B B A$. Background subtraction was obtained performing $A-B$ and $B-A$ image differences, obtaining four positive aperture images. The spectrum was extracted for each differential image in a standard way using the IRAF task apall. Then, in order to remove cosmic rays, the four extracted spectra were combined together. Finally, a telluric standard star was used to correct the target spectrum for the atmospheric transmission.

In producing the joint APM 08279+5255 UV-to-optical spectrum, we compared the restframe UV flux with a coeval spectrum taken in April 2011 at the Asiago observatory (Italy) for a reverberation-mapping campaign of luminous quasars (Trevese et al. 2007, 2014; Saturni et al. 2016). We noted that the TNG UV flux level obtained from standard-star calibration was a factor $\sim 1.1$ lower than the Asiago spectrum, which was acquired together with a reference star within a wide $\left(8^{\prime \prime}\right)$ slit in order to do not generate differential light losses and be therefore able to construct meaningful light curves (see e.g., Kaspi et al. 2007, for a discussion). We suspected that a light loss happened in acquiring the TNG/DOLoRes spectrum due to a seeing-limited observation rather than diffraction-limited (seeing at La Palma site of up to $\sim 1^{\prime \prime} .5$, to be compared with the $1^{\prime \prime}$ slit width). Additionally, no IR photometric standard stars were available during the NICS observing night. Therefore, we decided instead to recalibrate the APM $08279+5255$ full spectrum to the photometry reported in Egami et al. (2000); Ojha et al. (2009).

First, we matched the spectral sections together by scaling them to the integrated fluxes computed in intervals around two fiducial wavelengths, specifically $\lambda \sim 9200 \AA$ for the match between $R$ and $I J$ bands and $\lambda \sim 14400 \AA$ for the match between $I J$ and $H K$ bands. The matching wavelengths were selected by visual inspection, in order to identify overlapping spectral regions relatively free from fringing and superposition of contiguous spectral orders. The flux-calibrated spectrum was finally obtained by normalizing the joint sections to the corresponding photometry of APM $08279+5255$ available in the literature (see Table 1; BVRI magnitudes are from Egami et al. 2000, $J H K_{\mathrm{s}} L^{\prime}$ magnitudes are from Ojha et al. 2009). To do so, we produced spectro-photometric points by integrating the joint spectrum over the bands listed in Table 1; then, we performed a 1st-order spline fit to the ratios between APM 08279+5255 literature magnitudes and the spectro-photometric points, and obtained the final spectrum by multiplying the joint spectrum by this spline. This approach does not introduce a significant amount of additional uncertainty on the spectral flux level, since the average photometric error $\Delta m_{\mathrm{ph}}$ listed in Table 1 corresponds to a flux error of $\sim 9 \times 10^{-17} \mathrm{erg} \mathrm{s}^{-1} \mathrm{~cm}^{-2}$, which is comparable to the average rms spectrum over the whole wavelength range. 


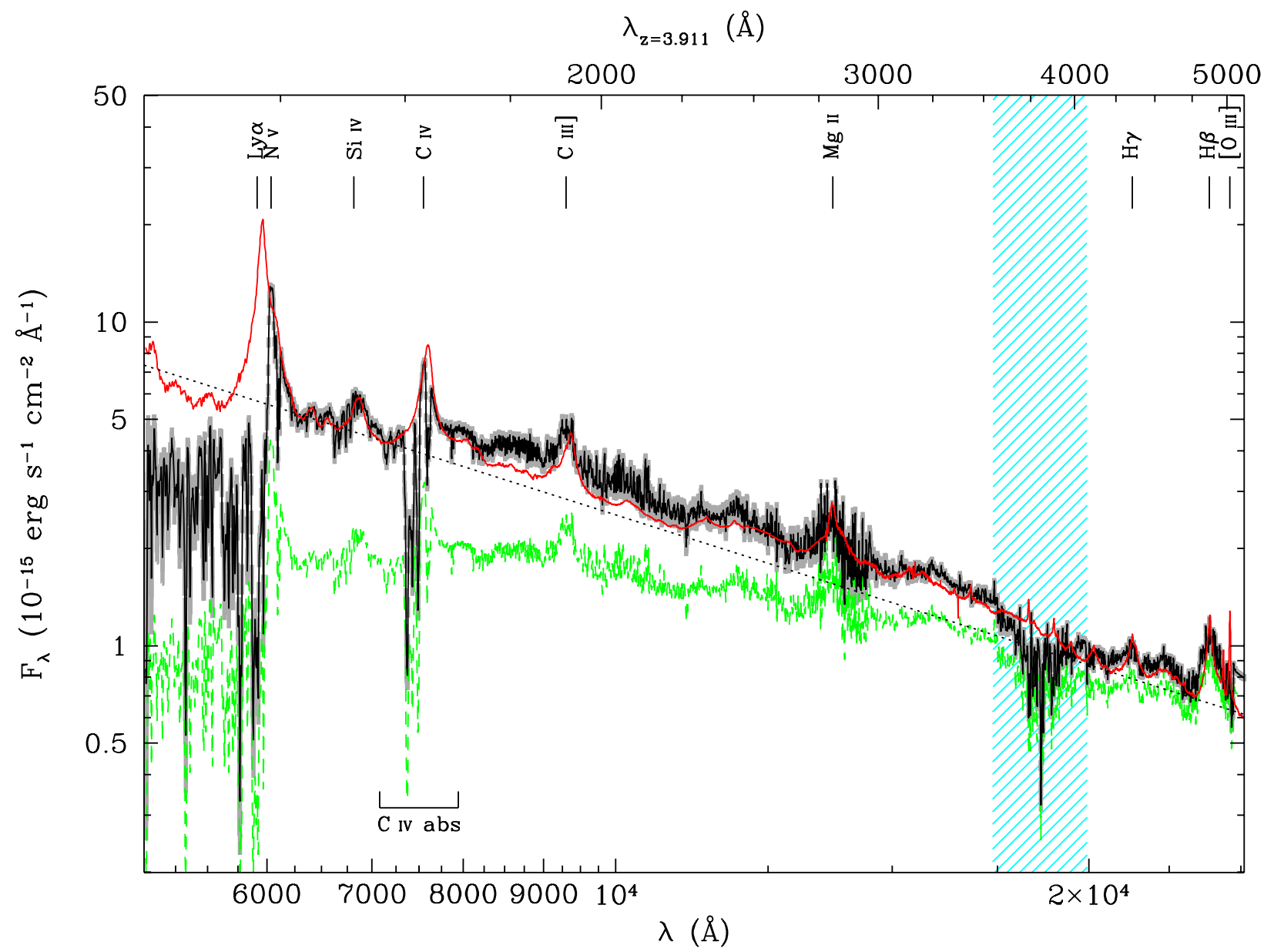

Fig. 1. Calibrated spectrum of APM $08279+5255$ before (green dashed line) and after dereddening (black solid line), with corresponding $1 \sigma$ uncertainty (gray band) - see Sect. 2. Superimposed to the data, the composite quasar template obtained by matching the Vanden Berk et al. (2001) and Glikman et al. (2006) templates around $\lambda \sim 3000 \AA$ in the restframe (red line) is shown. The continuum level is marked as a black dotted line with slope -1.54. A residual telluric absorption is still present between $\lambda \sim 17500 \AA$ and $20000 \AA$ (cyan shaded area), hence this spectral interval is excluded from the present analysis.

We checked that the magnitudes of APM $08279+5255$ used in the recalibration were measured in epochs in which the quasar continuum is not varying. The near-infrared magnitudes are taken at $M J D=51089$ (Egami et al. 2000), whereas the optical photometry is measured in runs between $M J D=53440$ and 54124 (Ojha et al. 2009). Comparing these epochs with those of the $R$-band photometric observations used to construct the light curve of APM $08279+5255$ in Trevese et al. (2013) and Saturni et al. (2016, see their Fig. 2), we note that they fall in periods during which the observer-frame optical flux of the quasar remains constant within $0.04 \mathrm{mag}$. Therefore, our photometry-based spectral recalibration is free of biases introduced by spectral variability.

In order to remove the dust reddening, the spectrum was further de-reddened according to a Small Magellanic Cloud (SMC) extinction law (Pei 1992) with $A_{\mathrm{V}}=0.6$ (Petitjean et al. 2000) at $z=1.062$, i.e., the probable redshift of the lensing galaxy (Ellison et al. 2004). Figure 1 shows the comparison of the joint restframe optical-to-UV spectrum of APM $08279+5255$ taken at TNG with a template obtained by matching the Vanden Berk et al. (2001) and the Glikman et al. (2006) SDSS broad-line quasar templates around $\lambda \sim 3000 \AA$ in the restframe, and normalized to match the observed flux at $1350 \AA$ in the restframe. In our procedure, we have not accounted for the intrinsic reddening of the host galaxy (e.g., Gallerani et al. 2010).
In general, BAL QSOs appear in fact to be more reddened by intrinsic dust with respect to normal quasars (see e.g., Richards et al. 2003, and refs. therein), with a BAL QSO fraction rising up to $\sim 40 \%$ in extremely reddened objects (e.g., Urrutia et al. 2009). However, the comparison of our de-reddened spectrum with the joint quasar template (which is in turn used in the following to compute the indexes of absorption for APM $08279+5255)$ provides no evidence for intrinsic dust reddening within the host galaxy. We thus prefer to consider only the reddening from the lensing galaxy, although we cannot rule out a possible contribution from the APM 08279+5255 host.

\section{Analysis of the UV and optical spectral feature}

\subsection{Absorption features}

The restframe UV-to-optical spectrum of APM $08279+5255$ is suitable to study the presence of broad absorption features other than those associated to C IV. To this purpose, we calculate the indexes of absorption most commonly used to identify and classify BAL QSOs, namely the "balnicity" index (BI; Weymann et al. 1991), the zero-velocity balnicity index $\left(\mathrm{BI}_{0}\right.$; Gibson et al. 2009) and the absorption index (AI; Trump et al. 2006). We performed this calculation for both the high- and low-ionization transitions that most frequently produce absorption in this class 
Table 1. Optical-to-near infrared Vega magnitudes of APM $08279+5255$ in the observer frame available in the literature.

\begin{tabular}{lcccc}
\hline \hline Band & $\lambda_{\mathrm{eff}}(\AA)$ & $m_{\mathrm{ph}}(\mathrm{mag})$ & $\Delta m_{\mathrm{ph}}(\mathrm{mag})$ & Ref. \\
\hline$B$ & 4380 & 18.827 & 0.017 & 1 \\
$V$ & 5450 & 16.448 & 0.012 & 1 \\
$R$ & 6410 & 15.353 & 0.014 & 1 \\
$I$ & 7980 & 14.608 & 0.012 & 1 \\
$J$ & 12200 & 13.340 & 0.030 & 2 \\
$H$ & 16300 & 12.650 & 0.030 & 2 \\
$K_{\mathrm{s}}$ & 21300 & 12.080 & 0.030 & 2 \\
$L^{\prime}$ & 34500 & 9.900 & 0.040 & 2 \\
\hline
\end{tabular}

References. (1) Ojha et al. (2009). (2) Egami et al. (2000).

of objects, that is, Si IV $\lambda 1400, \mathrm{C}$ IV $\lambda 1549, \mathrm{Al}$ III $\lambda 1860$, and Mg II $\lambda 2800$.

The mathematical expression of the $\mathrm{BI}, \mathrm{BI}_{0}$ and $\mathrm{AI}$ can be generalized into an integral quantity $I(\mathbf{k})$, where $\mathbf{k}=\left(k_{1 \rightarrow 5}\right)$ represents a set of parameters that define the integration limits, the minimal depth and the minimal velocity width of the absorption. We call $I(\mathbf{k})$ the generalized index of absorption, and define it as follows:

$I(\mathbf{k})=-\int_{-3000 k_{1}}^{-\left(25+4 k_{2}\right) \times 1000}\left[1-\frac{f(v)}{0.1 k_{3}}\right] C\left(k_{4}, k_{5}\right) \mathrm{d} v$,

where $f(v)$ is the normalized QSO flux in the velocity space, and $C\left(k_{4}, k_{5}\right)$ a constant assuming unitary value over absorbed regions in which the integrand function is positive, provided that the integrand itself remains greater than $0.1 k_{4}$ in contiguous portions of the absorption trough at least $1000 k_{5} \mathrm{~km} \mathrm{~s}^{-1}$ wide (otherwise, $C=0$ ). Within this scheme, the set $\mathbf{k}$ uniquely identifies each index of absorption: $\mathbf{k}_{\mathrm{BI}}=(1,0,9,0,2)$, $\mathbf{k}_{\mathrm{BI}_{0}}=(0,0,9,3,2)$, and $\mathbf{k}_{\mathrm{AI}}=(0,1,10,1,1)$. To compute these indexes, we normalize the dereddened APM 08279+5255 spectrum to the joint quasar template from Vanden Berk et al. (2001) and Glikman et al. (2006) shown in Fig. 1, then evaluating the $\mathrm{BI}, \mathrm{BI}_{0}$, and $\mathrm{AI}$ according to Eq. (1).

The formal error $\sigma_{I}^{2}(\mathbf{k})$ associated to $I(\mathbf{k})$ is connected to the rms error on the flux $\sigma_{f}(v)$ by

$\sigma_{I}^{2}(\mathbf{k})=-\int_{-3000 k_{1}}^{-\left(25+4 k_{2}\right) \times 1000}\left[\frac{\sigma_{f}(v)}{0.1 k_{3}}\right]^{2} C\left(k_{4}, k_{5}\right) \mathrm{d} v$,

although the real uncertainty is usually dominated by systematics in the continuum placement (Trump et al. 2006). Therefore, we adopt a Monte Carlo simulative approach to give a proper evaluation of the uncertainties associated to each nonzero index, or provide fiducial upper limits. Accordingly, we alter the spectrum by adding random noise with Poissonian distribution to each spectral bin, which is assumed to be the mean value of the noise distribution at its wavelength. We then recompute $I(\mathbf{k})$ on this altered spectrum, iterating the process $10^{3}$ times to reach statistical significance. Finally, we take the standard deviation of the $I(\mathbf{k})$ posterior distribution as the uncertainty to be associated to a nonzero index on the true spectrum. In case of null indexes, we set this standard deviation as the upper limit on the absorption strength. This procedure succeeds in producing fiducial uncertainties or upper limits for all the indexes of absorption but the $\mathrm{BI}_{0}$ for $\mathrm{Si}$ IV, $\mathrm{Al}$ III, and $\mathrm{Mg}$ II. This can be explained in terms of the conservative definition of $\mathrm{BI}_{0}$ given by Gibson et al. (2009), which requires deep absorption features to produce a positive value of this index.
Table 2. APM $08279+5255$ indexes of absorption associated to the main BAL transitions in quasars.

\begin{tabular}{lccc}
\hline \hline Transition & $\mathrm{BI}\left(\mathrm{km} \mathrm{s}^{-1}\right)$ & $\mathrm{BI}_{0}\left(\mathrm{~km} \mathrm{~s}^{-1}\right)$ & $\mathrm{AI}\left(\mathrm{km} \mathrm{s}^{-1}\right)$ \\
\hline Si IV & $<70$ & - & $<230$ \\
C IV & $3370 \pm 200$ & $2430 \pm 300$ & $4210 \pm 240$ \\
Al III & $<10$ & - & $<110$ \\
Mg II & $<170$ & - & $<490$ \\
\hline
\end{tabular}

Notes. Locations marked with "__" indicate indexes for which a reliable upper limit cannot be provided.

Figure 2 shows the normalized spectral regions around $\mathrm{Si}$ IV, $\mathrm{C}$ IV, $\mathrm{Al}$ III, and $\mathrm{Mg}$ II. The values of $\mathrm{BI}, \mathrm{BI}_{0}$, and $\mathrm{AI}$ for each transition are listed in Table 2, along with the associated uncertainty. We consider significant only absorption detected at a confidence level $>3 \sigma$. The only absorption feature that satisfies this criterion is the known BAL associated to C IV. However, when observed at high resolution, this absorption structure reveals to be not a single trough, but rather a complex system with two true C IV BALs at $v \sim-9750$ and $\sim-4500 \mathrm{~km} \mathrm{~s}^{-1}$ separated by narrow absorptions located around $v \sim-8500 \mathrm{~km} \mathrm{~s}^{-1}$ (Srianand \& Petitjean 2000). Variability studies suggest that this narrow-absorption complex is nevertheless associated with $\mathrm{C}$ IV outflows, since it shows a variability pattern very similar to the $\mathrm{C}$ IV BAL (see e.g., Fig. 6 of Trevese et al. 2013). In this case the integral in Eq. (1) is calculated across the whole spectral range $\lambda \lambda 1400-1550$, hence including all the features and giving values in agreement with those obtained by Trevese et al. (2013) for the equivalent width (EW) of the total absorption (from $\sim 2300$ to $\sim 4800 \mathrm{~km} \mathrm{~s}^{-1}$ ). In addition, the upper limits to the $\mathrm{Si}$ IV absorption strength reflect the sampling of the multiple narrow absorption identified by Ellison et al. (2004) as intervening C IV, Si IV, and Mg II features (EW between $\sim 90$ and $\sim 140 \mathrm{~km} \mathrm{~s}^{-1}$; see their Table 2 and Fig. 2). With respect to the low-ionization transitions, the Al III spectral region is affected by fringing in the 8300-9000 $\AA$ range that artificially increases the flux level with respect to the reference quasar template, thus preventing a reliable estimate of the Al III absorption strength. The low-significance $\mathrm{Mg}$ II feature, composed by two troughs respectively at $v \sim 0$ (not sampled by the BI) and $\sim-2 \times 10^{4} \mathrm{~km} \mathrm{~s}^{-1}$, is between approximately ten and twenty times weaker than the $\mathrm{C}$ IV BAL when the AI or BI are used. Furthermore, any firm conclusion about the presence of $\mathrm{Mg}$ II BALs is prevented by the lowsensitivity gap between the bands $J$ and $H$. Therefore, we classify APM 08279+5255 as a high-ionization BAL QSO (HiBAL) due to the lack of unambiguous low-ionization absorption features.

The most common class of BAL QSOs ( $~ 85 \%$ of the BAL QSO population; e.g., Farrah et al. 2007) is that of the HiBALs. In particular, BALs associated with C IV represent the most common absorption troughs found in quasars, and are widely used to study the evolution of the BAL QSO population with cosmic time (Hewett \& Foltz 2003; Reichard et al. 2003; Knigge et al. 2008; Gibson et al. 2009; Allen et al. 2011) as well as to characterize the ensemble absorption variability timescales (Barlow 1993; Lundgren et al. 2007; Gibson et al. 2008, 2010; Capellupo et al. 2011, 2012, 2013; Filiz Ak et al. 2014). For instance, the time variability of the APM $08279+5255 \mathrm{C}$ IV BAL has been studied in detail in Trevese et al. (2013) and Saturni et al. (2014, 2016), spanning a time interval of $\sim 19 \mathrm{yr}$ in the observer frame (i.e., $\sim 3.9 \mathrm{yr}$ in the restframe). The discovery of significant lowionization troughs would have been extremely interesting to, for 


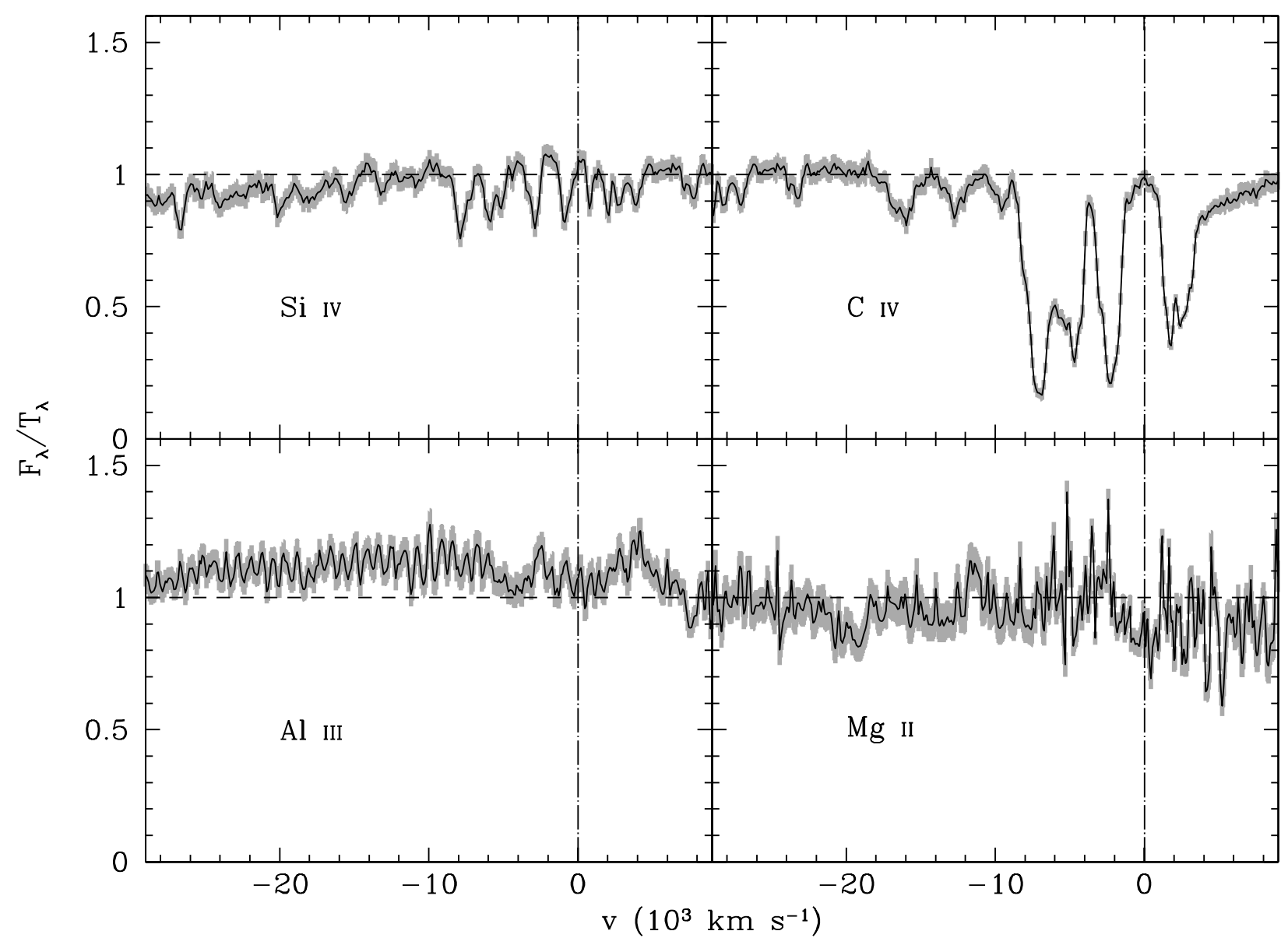

Fig. 2. Flux of APM 08279+5255 normalized to the Vanden Berk et al. (2001) and Glikman et al. (2006) joint quasar template. The spectral regions around the Si IV emission (top left panel), the $\mathrm{C}$ IV emission (top right panel), the $\mathrm{Al} \mathrm{III} \mathrm{emission} \mathrm{(bottom} \mathrm{left} \mathrm{panel)} \mathrm{and} \mathrm{the} \mathrm{Mg}$ II emission (bottom right panel) are shown. In all panels, the velocity scale is relative to the systemic redshift $z=3.911$ derived from the $\mathrm{CO}(4-3)$ and $\mathrm{CO}(9-8)$ emission lines (Downes et al. 1999). As a guidance, the zero-velocity position (dot-dashed line) and the normalized flux level (dashed line) are indicated.

example, unveil a possible transition between an obscured AGN phase and a normal quasar, as suggested by Farrah et al. (2007, 2012) for the case of ultraluminous IR galaxies (ULIRGs) such as APM 08279+5255 (Rowan-Robinson 2000). Nevertheless, the simultaneous presence of an UFO, a BAL and a molecular outflow in its spectrum makes APM $08279+5255$ similar to the local quasar and ULIRG Mrk 231 (Feruglio et al. 2015), configuring this object as one of the best potential targets to investigate multiphase outflows at higher redshifts and extreme energetic regimes (see e.g., Cicone et al. 2018).

\section{2. $\mathrm{Mg} ॥, \mathrm{H} \beta$, and [O III] emission lines}

In order to study the properties of the $\mathrm{Mg}$ II, $\mathrm{H} \beta$, and [O III] emission lines of APM $08279+5255$, we performed a spectral analysis of the two regions corresponding to the $\mathrm{H} \beta+\left[\mathrm{O}_{\text {III }}\right]$ (restframe wavelength range $\sim 3990-5040 \AA$, which also includes the $\mathrm{H} \gamma \lambda 4340$ emission) and $\mathrm{Mg}$ II (restframe wavelength range $\sim 2200-3500 \AA$ ). The analysis was done by using custom IDL processing scripts, based on the IDL package MPFIT (Markwardt 2009). The emission lines and the continuum emission were fitted together, by minimizing the $\chi^{2}$. Figure 3 shows a magnification out of such spectral regions, which are characterized by strong $\mathrm{Fe}$ II emission producing a complex pseudo-continuum close to $\mathrm{H} \beta$ and $\mathrm{Mg}$ II.
As a first step, we tried to account for this Fe II-related emission in the restframe optical range by including the typical observational Fe II templates from Boroson \& Green (1992), Véron-Cetty et al. (2004) and Tsuzuki et al. (2006) in the fits. We also considered the library of CLOUDY Fe II synthetic spectral templates presented in Bischetti et al. (2017) for hyper-luminous Type I quasars. In the fit, each template was convolved with a Gaussian whose width was free to vary, in order to account for the velocity dispersion of the gas. However, none of these templates was able to reproduce the Fe II spectral features observed in APM $08279+5255$. Furthermore, we also found that the observational Fe II template from Vestergaard \& Wilkes (2001) fails to reproduce the $\mathrm{Fe}$ II emission in the spectral region around $\mathrm{Mg}$ II. This failure might be partly due to the presence of telluric features in the near-IR spectra limiting the spectral windows used to anchor the fit. However, it is more likely that APM 08279+5255, being an exceptional object, shows an intrinsic difference in the $\mathrm{Fe}$ II emission properties, as the relative intensities of the main $\mathrm{Fe}$ II emission blends do not match any template from the adopted library. Furthermore, we also tried to simultaneously fit two different Fe II templates with independent velocity dispersion, as done in Vietri et al. (2018) in case of hyper-luminous quasars similar to APM $08279+5255$ with strong Fe II emission. However, this did not result in an improvement. Therefore, we fitted the most prominent $\mathrm{Fe}$ II features in the spectrum by means of 


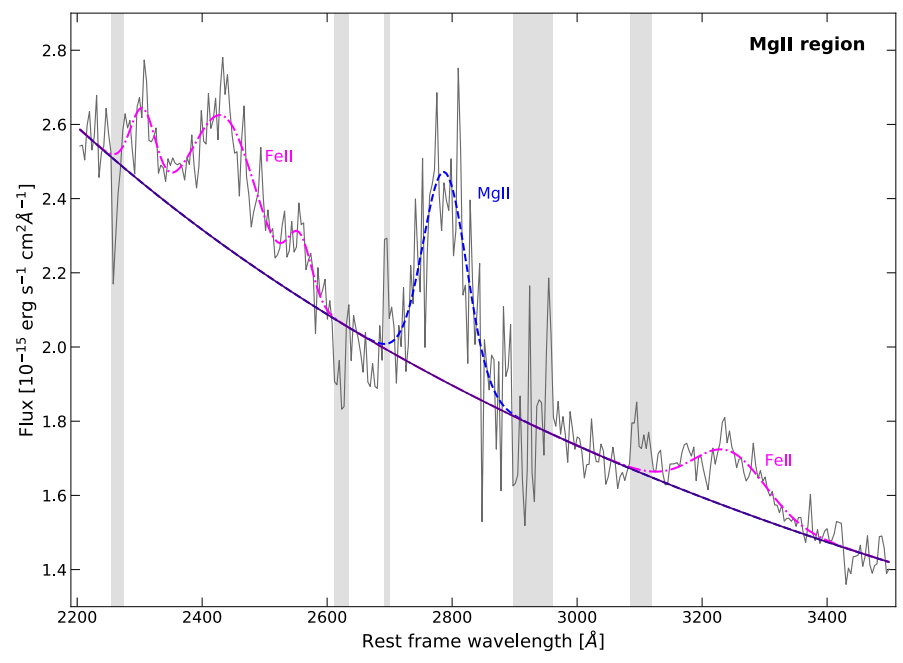

(a)

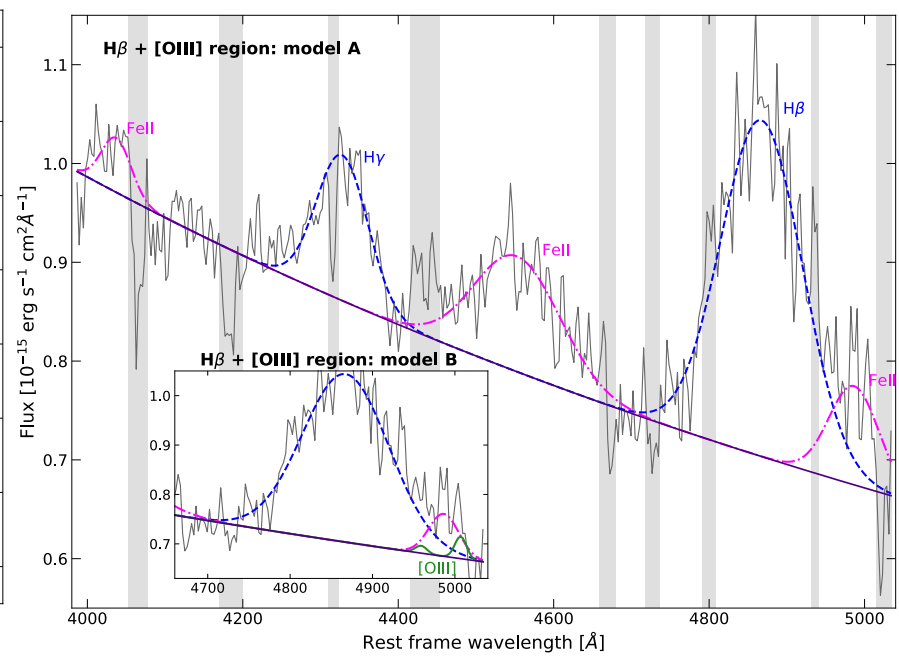

(b)

Fig. 3. NICS spectrum of APM $08279+5255$ corresponding to the Mg II and $\mathrm{H} \beta+[\mathrm{O}$ III] spectral regions. Specifically, panel $a$ shows the best fit description of the restframe $\sim 2200-3400 \AA$ band. Panel $b$ and its inset show the best-fit description of the restframe $\sim 3990-5040 \AA$ band according to models A and B, respectively. In all panels, a purple solid line marks the power-law quasar continuum. Blue dashed curves refer to $\mathrm{Mg}$ II, $\mathrm{H} \gamma$, and $\mathrm{H} \beta$ emission lines, whereas the Fe II emission is plotted as magenta dot-dashed lines. In model $\mathrm{B}$, the green curve refers to the [O III] emission. Gray bands indicate the regions excluded from the fit because of the presence of telluric features.

multiple Gaussian components. We tried to limit the dependence of the resulting line parameters on the adopted model by using a minimum number of $\mathrm{Fe}$ II components: in other words, we checked that adding another Gaussian component did not lead to a significant improve of the $\chi^{2}$. However, a modest degeneracy between subtle Fe II emission and the other emission lines might still be present.

The spectral fitting procedure in the $\mathrm{H} \beta+[\mathrm{O}$ III] range is particularly challenging due to the combination of (i) a limited spectral coverage redward $5007 \AA$, (ii) the fact that these lines fall very close to the edge of the NICS wavelength coverage, which is affected by telluric absorption, and (iii) the lack of a clear emission feature at the wavelengths expected for the $[\mathrm{O} \mathrm{III}]$ doublet. We thus tested two spectral models in order to infer the properties of any subtle [O III] emission. The first model (model A hereafter) only considers the presence of $\mathrm{Fe}$ II emission redward the $\mathrm{H} \beta$ emission, while the second model (model B) accounts for the presence of both [O III] and Fe II in this spectral region. Specifically, model A includes a Gaussian component with FWHM free to vary in order to account for the Fe II emission at $4861<\lambda<5000 \AA$ in the restframe; the upper bound was set taking into account the NICS spectral resolution of $\sim 40 \AA$ at these wavelengths in order to not overlap with spectral regions involving possible [O III] emission at $5007 \AA$.

Model B is similar to model A, but also includes two Gaussian components to fit the [O III] $\lambda \lambda 4959,5007 \AA$ doublet with a fixed $F W H M=1000 \mathrm{~km} \mathrm{~s}^{-1}$, which is a typical upper limit to the width of emission lines associated to the narrowline region. The centroids of the [O III] doublet components were fixed to $5007 \AA$ and $4959 \AA$ in the restframe, and the ratio of their normalizations was fixed at 1:3. Furthermore, both models include $n$ total four Gaussian components to fit the $\mathrm{H} \beta$ and $\mathrm{H} \gamma$ broad emissions, the strong $\mathrm{Fe}$ II emission features centered at $\sim 4050 \AA$ and $\sim 4550 \AA$, and a power law to parameterize the continuum emission. Apart from the [O III] doublet, the velocity offset between all Gaussian components are free to vary. Both models are shown in Fig. $3 \mathrm{~b}$.
The restframe main spectral parameters, derived from the different models applied to the NICS data, are shown in Table 3. The $\mathrm{H} \beta$ emission is well reproduced by a broad Gaussian profile, with a $F W H M_{H \beta} \sim 7000-7400 \mathrm{~km} \mathrm{~s}^{-1}$, that appears to be slightly redshifted $\left(\lambda_{\mathrm{H} \beta} \sim 4868 \AA\right.$, i.e., $\left.\sim 410 \mathrm{~km} \mathrm{~s}^{-1}\right)$ with respect to the systemic redshift $z=3.911$ inferred from CO lines (Downes et al. 1999); however, given the dispersion error of $\sim 8 \AA$ in the restframe associated to the grism, the position of the $\mathrm{H} \beta$ emission peak is still consistent with the assumed systemic redshift. The addition of the [O III]related components in model B yields a decrease of $\Delta \chi^{2}=12$ for one additional free parameter (i.e., the [O III] $\lambda 5007 \AA$ normalization) compared to model $\mathrm{A}$, which represents a statistical improvement at $98.7 \%$ confidence level according to an $F$-test. This suggests that a weak [O III] emission with $F_{\text {[OIII] }}(5007 \AA)=(1.8 \pm 0.7) \times 10^{-15} \mathrm{erg} \mathrm{s}^{-1} \mathrm{~cm}^{-2}$, corresponding to an [O III]-to-H $\beta$ flux ratio $F_{[\mathrm{OIII}]} / F_{\mathrm{H} \beta}=0.04$, can still be present in the optical spectrum of APM $08279+5255$. Such an [O III] weakness is consistent with the detection of strong $\mathrm{Fe}$ II emission, according to Eigenvector 1 (e.g., Boroson \& Green 1992; Shen \& Ho 2014). However, we stress that any firm conclusion on the properties of [O III] emission in APM 08279+5255 is hampered by the low $\mathrm{S} / \mathrm{N}$ and limited spectral coverage of the NICS data.

As for the fit to the spectral data in the $\mathrm{Mg}$ II emission region, we used a model consisting of one Gaussian component that accounts for the $\mathrm{Mg}$ II line, four Gaussian components to fit the main Fe II emission features centered respectively at $\sim 2300 \AA$, $\sim 2450 \AA, \sim 2550 \AA$ and $\sim 3200 \AA$ in the restframe, and a power law for the underlying continuum (see Fig. 3a). Such a fit yields a good description of the spectrum with an associated reduced $\chi^{2}=1.45$. The best-fit value for the FWHM of the Mg in broad emission line is $9200_{-440}^{+610} \mathrm{~km} \mathrm{~s}^{-1}$. Remarkably, we found that the $\mathrm{Mg}$ II emission centroid is located at $\lambda_{\mathrm{MgII}} \sim 2789 \AA$ blueshifted by $11 \AA\left(1180 \pm 430 \mathrm{~km} \mathrm{~s}^{-1}\right)$ with respect to the expected value at the systemic redshift. This blueshift is a factor of approximately two larger than the restframe dispersion error associated to the 
Table 3. Spectral fit results derived for the $\mathrm{H} \beta+[\mathrm{O}$ III $]$ and $\mathrm{Mg}$ II regions of APM $08279+5255$.

\begin{tabular}{|c|c|c|c|}
\hline \multirow[t]{2}{*}{ Parameter } & \multicolumn{2}{|c|}{$\mathrm{H} \beta+[\mathrm{O}$ III $]$ region } & \multirow[t]{2}{*}{ Units } \\
\hline & $\mathrm{A}$ & $\mathrm{B}$ & \\
\hline$\chi^{2} / N_{\text {d.o.f. }}$ & $3863 / 2012$ & $3851 / 2011$ & \\
\hline $\mathrm{FWHM}_{\mathrm{H} \beta}$ & $6990 \pm 460$ & $7360 \pm 430$ & $\mathrm{~km} \mathrm{~s}^{-1}$ \\
\hline$\lambda_{\mathrm{H} \beta}$ & $4866 \pm 7$ & $4868 \pm 7$ & $\AA$ \\
\hline$F_{\text {[OIII] }}(5007 \AA)$ & - & $1.8 \pm 0.7$ & $10^{-15} \mathrm{erg} \mathrm{s}^{-1} \mathrm{~cm}^{-2}$ \\
\hline$\lambda \mathrm{L}_{\lambda}(5100 \AA)$ & $5.1 \pm 1.1$ & $5.1 \pm 1.2$ & $10^{47} \mathrm{erg} \mathrm{s}^{-1}$ \\
\hline $\mathrm{H} \beta$ velocity shift & \multicolumn{2}{|c|}{$\lesssim 840$} & $\mathrm{~km} \mathrm{~s}^{-1}$ \\
\hline$\underline{\text { Parameter }}$ & \multicolumn{2}{|c|}{ Mg II region } & Units \\
\hline$\chi^{2} / N_{\text {d.o.f. }}$ & \multicolumn{2}{|c|}{$3186 / 2197$} & \\
\hline FWHM $_{\mathrm{MgII}}$ & \multicolumn{2}{|c|}{$9200_{-440}^{+610}$} & $\mathrm{~km} \mathrm{~s}^{-1}$ \\
\hline$\lambda_{\mathrm{MgII}}$ & \multicolumn{2}{|c|}{$2789 \pm 4$} & $\AA$ \\
\hline$\lambda \mathrm{L}_{\lambda}(3000 \AA)$ & \multicolumn{2}{|c|}{$7.9 \pm 1.9$} & $10^{47} \mathrm{erg} \mathrm{s}^{-1}$ \\
\hline Mg II velocity shift & \multicolumn{2}{|c|}{$-1180 \pm 430$} & $\mathrm{~km} \mathrm{~s}^{-1}$ \\
\hline
\end{tabular}

grism resolution in this spectral range, and is also approximately two times smaller than the blueshift of $2500 \pm 40 \mathrm{~km} \mathrm{~s}^{-1}$ measured for the $\mathrm{C}$ IV emission (and valid for other high-ionization emission lines such as N v, Si IV and C III]; e.g., Irwin et al. 1998) from the $R$-band spectrum taken with the high-resolution spectrograph STIS on board of HST (Lewis et al. 2002b). We show this blueshift dependence on wavelength in Fig. 4, where the centroid shifts measured for $\mathrm{C}$ IV, $\mathrm{Mg}$ II, and $\mathrm{H} \beta$ broad emission lines are compared. This figure highlights that the $\mathrm{Mg}$ II emission line can be affected by a strong blueshift in the same way as high-ionization features. This can therefore bias the single-epoch SMBH mass estimate based on this low-ionization transition in case of highly-accreting AGN (e.g., Marziani et al. 2013). In particular, $\mathrm{Mg}$ II is extensively used to measure the SMBH mass of high-luminosity quasars at $z>5$, due to the fact that $\mathrm{H} \beta$ line is no longer observable in the $K$ band.

Previous studies found the [O III] weakness to be associated with broad blueshifted C IV emission in samples of Type 1 luminous quasars (e.g., Netzer et al. 2004). In their study of the optical-to-UV spectra of WISE/SDSS selected hyper-luminous quasars (WISSH; e.g., Bischetti et al. 2017), Vietri et al. (2018) have indeed found that $\sim 70 \%$ of them exhibit very weak [O $\mathrm{III}]$ emission $(<5 \AA)$ and largely blueshifted $\left(\approx 2000-8000 \mathrm{~km} \mathrm{~s}^{-1}\right)$ $\mathrm{C}$ IV emission with restframe EWs $\lesssim 20 \AA$. They interpreted these properties in terms of a steep UV-to-X-ray continuum in luminous quasars coupled to a face-on view of the continuum source. The former property leads to an efficient line-driving acceleration mechanism for broad-line region (BLR) winds (Proga \& Kallman 2004; Wu et al. 2009; Risaliti \& Elvis 2010; Richards et al. 2011), while the latter implies an observed small EW of the [O III] emission line (see Bisogni et al. 2017). The reconstruction of the $\mathrm{C}$ IV line profile on the APM $08279+5255 \mathrm{HST} / \mathrm{STIS}$ spectrum made by Saturni et al. (2016) allows us to compute a C IV restframe EW of $24 \pm 2 \AA$. This value is in agreement with the $\mathrm{C}$ IV EWs commonly measured in other very luminous quasars with weak [O III] and large $\mathrm{C}$ IV blueshift.

\section{Single-epoch black hole mass estimates}

The TNG observations of APM $08279+5255$ allow us to provide the virial single-epoch black hole mass $M_{\mathrm{BH}}$ using $\mathrm{Mg}$ II and $\mathrm{H} \beta$ emission lines. These lines are much more reliable

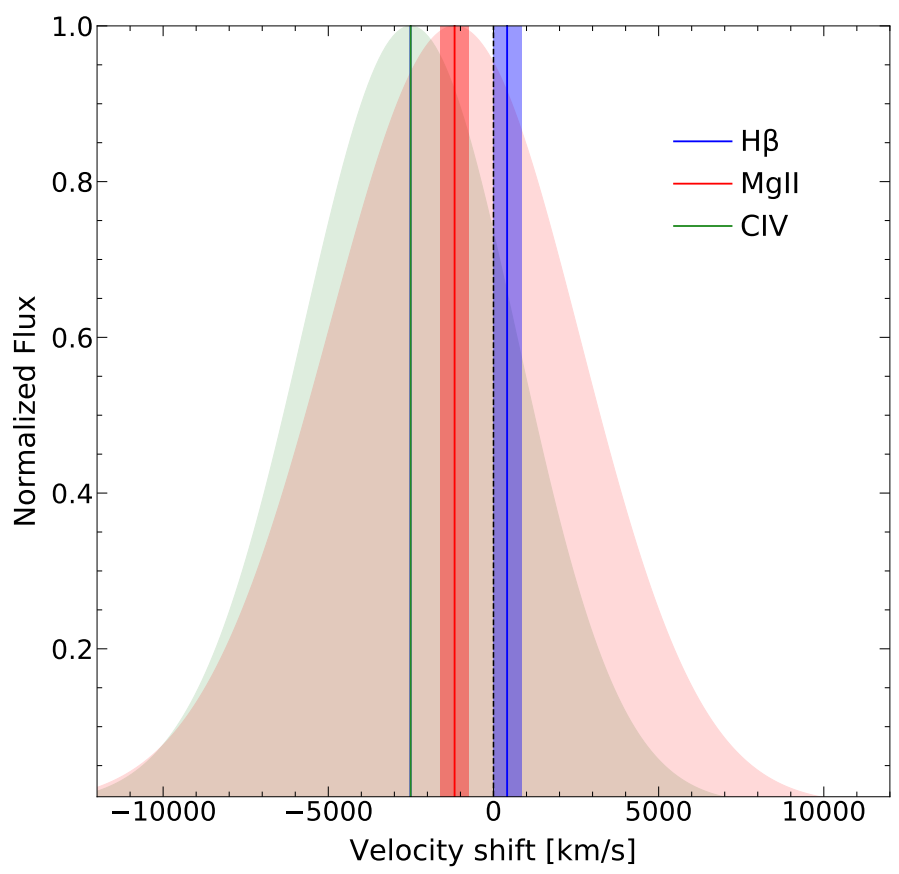

Fig. 4. Velocity shifts of the $\mathrm{H} \beta$ (blue), $\mathrm{Mg}$ II (red) and $\mathrm{C}$ IV (green) lines with respect to $z=3.911$. The centroids of the best fit Gaussian profiles resulting from our analysis ( $\mathrm{H} \beta$ and $\mathrm{Mg}$ II) and Saturni et al. (2016, C IV, based on high-resolution HST/STIS data) are indicated by vertical lines with the corresponding uncertainties (shaded vertical bands).

proxies of the BLR dynamics than C IV, whose profile is potentially affected by nonvirial motion of the emitting gas (Baskin \& Laor 2005; Shen \& Liu 2012; Vietri et al. 2018). Furthermore, in the case of APM $08279+5255$ the presence of strong BAL systems introduces additional uncertainty in the fit of the intrinsic $\mathrm{C}$ IV line profile. A direct measurement of $M_{\mathrm{BH}}$ based on C IV and Si IV reverberation mapping (RM; e.g., Peterson 1997) was provided by Saturni et al. (2016), who analyzed the HST/STIS spectrum. They were, therefore, able to accurately recover the C IV emission profile without the contamination of telluric features associated to the Fraunhofer A band on the red wing of the line, deriving a FWHM of $7480 \pm 70 \mathrm{~km} \mathrm{~s}^{-1}$ and, in turn, a $\log M_{\mathrm{BH}} / M_{\odot}=10.00_{-0.05}^{+0.07}$. The same RM-based value ${ }^{1}$ of $M_{\mathrm{BH}}$ was also obtained for the Si IV line, strengthening an estimate that could be affected by nonvirial components as in the case of C IV.

In our calculations, we have adopted the single-epoch relations derived by Bongiorno et al. (2014) for $\mathrm{Mg}$ II and $\mathrm{H} \beta$, using the FWHMs and reddening-corrected monochromatic luminosities listed in Table 3. These relations can be expressed in the form:

$\log \left(\frac{M_{\mathrm{BH}}}{M_{\odot}}\right)=a+0.5 \log \left[\frac{\lambda L_{\lambda}(3000 \AA / 5100 \AA)}{10^{44} \mathrm{erg} \mathrm{s}^{-1}}\right]+2 \log \left(\frac{\mathrm{FWHM}_{\mathrm{MgII} / \mathrm{H} \beta}}{1000 \mathrm{~km} \mathrm{~s}^{-1}}\right)$,

with the parameter $a=6.6$ for $\mathrm{Mg}$ II and 6.7 for $\mathrm{H} \beta$ respectively. In addition to the estimates of $M_{\mathrm{BH}}$ based on the $\mathrm{H} \beta$ and $\mathrm{Mg}$ II from the TNG spectrum, we also derive an additional

\footnotetext{
1 We note that this result strictly holds only assuming a form factor $f=5.5$ (Onken et al. 2004) for the high-ionization BLR. Other choices of the value of $f$ based on different calibrations of the BH mass-tovelocity dispersion relations (Graham et al. 2011; Park et al. 2012; Grier et al. 2013; Shen \& Ho 2014) or on the study of BLR geometry (Pancoast et al. 2014, 2015) are possible.
} 
Table 4. SMBH mass and Eddington ratio of APM $08279+5255$ derived from $\mathrm{H} \beta, \mathrm{Mg}$ II and $\mathrm{C}$ IV broad emission lines, as a function of the magnification parameter $\mu$.

\begin{tabular}{lccc}
\hline \hline \multicolumn{4}{c}{$\log \left(M_{\mathrm{BH}} / M_{\odot}\right)$} \\
\hline Transition & $\mu=1$ & $\mu=4$ & $\mu=100$ \\
\hline $\mathrm{H} \beta$ & $10.29 \pm 0.32$ & $9.99 \pm 0.32$ & $9.29 \pm 0.32$ \\
Mg II & $10.45 \pm 0.34$ & $10.14 \pm 0.34$ & $9.45 \pm 0.34$ \\
C IV & $10.54 \pm 0.31$ & $10.22 \pm 0.31$ & $9.48 \pm 0.31$ \\
\hline \multicolumn{4}{c}{$\lambda_{\text {Edd }}$} \\
\hline Transition & $\mu=1$ & $\mu=4$ & $\mu=100$ \\
\hline $\mathrm{H} \beta$ & $1.1 \pm 0.8$ & $0.5 \pm 0.4$ & $0.11 \pm 0.08$ \\
$\mathrm{Mg}$ II & $0.8 \pm 0.6$ & $0.4 \pm 0.3$ & $0.08 \pm 0.05$ \\
$\mathrm{C}$ IV & $0.7 \pm 0.4$ & $0.4 \pm 0.2$ & $0.08 \pm 0.05$ \\
\hline
\end{tabular}

Notes. The C IV-based estimates rely on the work by Saturni et al. (2016). The Eddington ratio has been computed using a bolometric luminosity derived according to Runnoe et al. (2012a,b).

one based on C IV. We use the Gaussian fit to the C IV emission from the HST/STIS spectrum performed by Saturni et al. (2016) and adopt the single-epoch relation from Vestergaard \& Peterson (2006) corrected according to the prescription on the line blueshift $\Delta v_{\mathrm{CIV}}$ for high-luminosity quasars (Coatman et al. 2017). This correction yields:

$$
\begin{aligned}
\log \left(\frac{M_{\mathrm{BH}}}{M_{\odot}}\right)= & 6.71+0.53 \log \left[\frac{\lambda L_{\lambda}(1350 \AA)}{10^{44} \mathrm{erg} \mathrm{s}^{-1}}\right] \\
& +2 \log \left(\frac{\mathrm{FWHM}_{\mathrm{CIV}}}{1000 \mathrm{~km} \mathrm{~s}^{-1}}\right) \\
& -2 \log \left[\alpha\left(\frac{\Delta v_{\mathrm{CIV}}}{1000 \mathrm{~km} \mathrm{~s}^{-1}}\right)+\beta\right],
\end{aligned}
$$

with $\alpha \approx 0.4$ and $\beta \approx 0.6$.

Table 4 shows the estimates of the $M_{\mathrm{BH}}$ of APM $08279+5255$ based on the different transitions. Due to the fact that APM $08279+5255$ is lensed by a foreground system which remains unobserved, at least two competing lens models have been proposed to explain the lack of a lens image. Egami et al. (2000) proposed a naked-cusp configuration in which the magnification at UV-to-optical wavelengths can rise up to $\sim 100$. Riechers et al. (2009) derived their lens model from the analysis of the $\mathrm{CO}$ emission region, finding a lower, almost achromatic magnification of approximately four. Accordingly, Table 4 lists the SMBH mass estimates as a function of different values of the magnification parameter: $\mu=1$ (i.e., no magnification), 4 and 100 respectively. The error associated to $\mathrm{M}_{\mathrm{BH}}$ includes in quadrature both the statistical uncertainties and intrinsic scatter in the single-epoch relations of $\sim 0.26 \mathrm{dex}$ (Vestergaard \& Peterson 2006; Shen et al. 2011; Bongiorno et al. 2014). Even in the most conservative case of $\mu=100$, the $M_{\mathrm{BH}}$ derived by considering the $\mathrm{H} \beta$ emission line is $>10^{9} M_{\odot}$, which indicates that APM $08279+5255$ harbors a SMBH at the heaviest end of the $M_{\mathrm{BH}}$ distribution.

In Table 4 we also report the estimate of the Eddington ratios $\lambda_{\text {Edd }}=L_{\text {bol }} / L_{\text {Edd }}$ based on these $M_{\mathrm{BH}}$ values, assuming a bolometric luminosity $L_{\mathrm{bol}}=2.7 \times 10^{48} \mathrm{erg} \mathrm{s}^{-1}$ before correcting for $\mu=4$ and 100 (which correspond to a true bolometric luminosity of $6.8 \times 10^{47} \mathrm{erg} \mathrm{s}^{-1}$ and $2.7 \times 10^{46} \mathrm{erg} \mathrm{s}^{-1}$ respectively).
We derive such a luminosity from the lensed monochromatic luminosity at $3000 \AA$ adopting the bolometric correction with nonzero intercept by Runnoe et al. (2012a,b):

$$
L_{\text {bol }}=0.75\left[10^{1.852} \cdot \lambda L_{\lambda}(3000 \AA)^{0.975}\right]
$$

We consider this estimate of $L_{\mathrm{bol}}$ as our fiducial value instead of deriving such a quantity from the fit of APM $08279+5255$ spectral energy distribution (SED), since we note that the SED itself is affected by a wavelenght-dependent magnification factor resulting from the contribution of emitting regions of different size. In this way, we are also consistent with the UV-to-optical luminosities used to evaluate the single-epoch SMBH mass of APM $08279+5255$.

Dong et al. (2009) reported the existence of a strong correlation between the EW of $\mathrm{Mg}$ II and $\lambda_{\mathrm{Edd}}$, i.e., $\mathrm{EW}_{\mathrm{MgII}} \propto \lambda_{\mathrm{Edd}}^{-0.4}$. Such an effect is possibly due to a decrease in the covering factor of the $\mathrm{Mg}$ II BLR at increasing $\lambda_{\mathrm{Edd}}$ (i.e., the number of $\mathrm{Mg}$ II emitting clouds decreases due to radiation-pressure blowing; see also Fabian et al. 2006; Marconi et al. 2008, 2009). We used this relation to obtain an independent estimate of $\lambda_{\text {Edd }}$ for APM $08279+5255$. The restframe EW derived from the TNG spectrum is $\mathrm{EW}_{\mathrm{MgII}}=27.1 \pm 1.5 \AA$, which corresponds to $\lambda_{\text {Edd }}=0.36_{-0.21}^{+0.51}$ taking into account the scatter of $0.38 \mathrm{dex}$ in $\lambda_{\text {Edd }}$ in the Dong et al. (2009) data. We note that the confidence interval of $\lambda_{\text {Edd }}$ obtained in this way is fully consistent with the values listed in Table 4 for the case of $\mu=4$, and only marginally consistent for the case of $\mu=100$. This suggests that a moderate lens magnification $\mu<100$ for APM $08279+5255$ can be favored over a more extreme value.

We can then derive an independent estimate of $\mu$ using the $\lambda_{\text {Edd }}$ obtained from the Dong et al. (2009) relation. In fact, from Eq. (3) we derive that $\lambda_{\text {Edd }} \propto \mu^{1-b}$, and hence

$\mu=\left[\frac{\lambda_{\mathrm{Edd}}^{\text {(obs) }}}{\lambda_{\text {Edd }}^{\text {(true) }}}\right]^{\frac{1}{1-b}}$,

where $b$ is the coefficient multiplying $\log \left(\lambda L_{\lambda}\right)$ in Eqs. (3) and (4) (0.5 for $\mathrm{H} \beta$ and $\mathrm{Mg}$ II from Bongiorno et al. 2014, and 0.53 for $\mathrm{C}$ IV from Vestergaard \& Peterson 2006). Therefore, a $\lambda_{\text {Edd }}^{\text {(true) }} \sim 0.36$ corresponds to $4.1 \lesssim \mu \lesssim 9.3$ (and, in turn, a bolometric luminosity $2.9 \times 10^{47} \lesssim L_{\text {bol }} \lesssim 6.7 \times$ $10^{47} \mathrm{erg} \mathrm{s}^{-1}$ and a black hole mass $6.5 \times 10^{9} \lesssim M_{\mathrm{BH}} \lesssim 1.5 \times$ $10^{10} M_{\odot}$ ) using as $\lambda_{\text {Edd }}^{\text {(obs) }}$ the values listed in the first column of Table 4 (i.e., the case of no magnification). Such an intermediate magnification factor is also in agreement with the upper limit of $\mu \lesssim 8.2$ derived by Saturni et al. (2016) from the RM. We also note that a lower limit on APM $08279+5255$ black hole mass $M_{\mathrm{BH}} \gtrsim 4 \times 10^{9} M_{\odot}$ has been derived by Hagino et al. (2017) through SED modeling. Using our fiducial luminosity and FWHM measurements for Mg II in Eq. (3) (see Table 3), we find that this limit is respected for $\mu \lesssim 50$.

\section{Summary and conclusions}

In this work, we have presented the quasi-simultaneous UV-tooptical spectrum of APM $08279+5255$ taken at TNG with the instruments DOLoRes and NICS. The presence of a UFO, a BAL and a molecular outflow in this object is of great interest to explore the properties of multiphase quasar winds at high redshifts and extreme luminosities with dedicated multiwavelength observations, in order to probe the possible presence of 
ongoing AGN feedback. This spectrum covers the previously unobserved region between $\mathrm{C}$ III] and [O $\mathrm{III}$ ], thus providing important constraints on the BAL classification, the SMBH mass, the Eddington ratio and the magnification factor in this high- $z$ quasar. Our main results can be summarized as follows:

- We tested the balnicity of APM $08279+5255$ for high- and low-ionization transitions. We computed the most commonly used indexes (BI, $\mathrm{BI}_{0}$, and $\mathrm{AI}$ ) of absorption for $\mathrm{Si}$ IV, $\mathrm{C}$ IV, $\mathrm{Al}$ III, and $\mathrm{Mg}$ II, confirming the BAL only for $\mathrm{C}$ IV and hence supporting a HiBAL rather than a LoBAL classification for APM $08279+5255$.

- The near-infrared NICS spectrum has allowed us, for the first time, to study the spectral regions corresponding to the $\mathrm{H} \beta+[\mathrm{O}$ III $]$ and $\mathrm{Mg}$ II emission lines in APM 08279+5255. The $\mathrm{H} \beta$ line profile shows a FWHM of $\sim 7400 \mathrm{~km} \mathrm{~s}^{-1}$ and a centroid consistent with the CO-based systemic redshift $z=3.911$. Conversely, the $\mathrm{Mg}$ II emission line $\left(F W H M \sim 9200 \mathrm{~km} \mathrm{~s}^{-1}\right)$ is characterized by a blueshift of $\sim 1200 \mathrm{~km} \mathrm{~s}^{-1}$, lower by a factor of approximately two than the blueshift of $\sim 2500 \mathrm{~km} \mathrm{~s}^{-1}$ measured for the $\mathrm{C}$ IV emission line. This result is in agreement with previous works that find larger blueshifts in high-ionization transitions (Richards et al. 2002; Baskin \& Laor 2005).

- We also investigated the presence of [O III] $\lambda \lambda 4959,5007 \AA$ emission in a spectral region very close to the red edge of the NICS spectrum and characterized by strong Fe II emission. Our best-fit model includes a low-significance [O III] component with $F_{\text {[OIII }}(5007 \AA) \sim(1.8 \pm 0.7) \times 10^{-15}$ $\mathrm{erg} \mathrm{s}^{-1} \mathrm{~cm}^{-2}$. This indicates that the $\left[\begin{array}{ll}\mathrm{O} & \mathrm{III}\end{array}\right]$ emission in APM $08279+5255$ is intrinsically weak with $F_{\text {[OIII] }} / F_{\mathrm{H} \beta}=0.04$, consistent with the prediction of Eigenvector 1 (Boroson \& Green 1992) of an anti-correlation between Fe II and [O III] emission. APM 08279+5255 therefore shares the properties of the [O III] and $\mathrm{C}$ IV emission lines observed in other sources lying at the bright end of the AGN luminosity function (Richards et al. 2011; Shen \& Ho 2014; Zuo et al. 2015; Marziani et al. 2016; Shen 2016; Vietri et al. 2018).

- We have been able to derive for the first time estimates of the $M_{\mathrm{BH}}$ in APM $08279+5255$ based on the $\mathrm{H} \beta$ and $\mathrm{Mg}$ II emission lines. These transitions have been found to provide a much more reliable measurement of $M_{\mathrm{BH}}$ in AGN than C IV. This is even more true in the case of APM $08279+5255$, whose $\mathrm{C}$ IV emission line is affected by BAL features. We find very large mass values $\left(\log M_{\mathrm{BH}} / M_{\odot} \gtrsim 9.3\right)$ for a magnification factor $\mu$ varying from 1 (i.e., no magnification) to 100 (Egami et al. 2000). A value of $\mu=4$ (Riechers et al. 2009) is compatible with the RM-based $M_{\mathrm{BH}}$ estimate of $\log M_{\mathrm{BH}} / M_{\odot} \sim 10$ given in Saturni et al. (2016). The $\mathrm{EW}_{\mathrm{MgII}}$-based estimate of $\lambda_{\mathrm{Edd}}$ according to the EW $-\lambda_{\mathrm{Edd}}$ relation by Dong et al. (2009) also suggests a moderate magnification factor $4.1 \lesssim \mu \lesssim 9.3$, corresponding to an intrinsic bolometric luminosity $2.9 \times 10^{47} \lesssim L_{\text {bol }} \lesssim 6.7 \times 10^{47} \mathrm{erg} \mathrm{s}^{-1}$ and a black hole mass $6.5 \times 10^{9} \lesssim M_{\mathrm{BH}} \lesssim 1.5 \times 10^{10} M_{\odot}$ ). This is in turn compatible with both the upper limit $\mu \lesssim 8.2$ found by Saturni et al. (2016) with RM, and the limit $\mu \lesssim 50$ derived from the single-epoch relations by adopting the minimal black hole mass $M_{\mathrm{BH}}=4 \times 10^{9} M_{\odot}$ inferred by Hagino et al. (2017) through APM 08279+5255 SED modeling.

Being taken 76 days apart (corresponding to only $\sim 15$ restframe days), the UV and optical spectral sections of APM 08279+5255 probe the same AGN state. This allowed us to study its physical properties consistently during a state of relatively constant
AGN emission. Indeed, this source has been found to vary in continuum, emission- and absorption-line intensity, with flux changes of up to $\sim 0.5 \mathrm{mag}$ (Trevese et al. 2013; Saturni et al. 2016). Therefore, multiband spectroscopic observations of APM $08279+5255$ must be quasi-simultaneous in order to overcome the lack of a common reference for a compatible flux calibration between spectra taken at different epochs. The possibility to observe APM 08279+5255 with the forthcoming James Webb Space Telescope (JWST) is hence of extreme interest, since the spectro-photometric capabilities of its instruments, simultaneously covering the wavelength range $\lambda \lambda 6000-2.8 \times 10^{5} \AA$ (Dorner et al. 2016; Labiano et al. 2016), may allow for this source the detailed study of the stratified BLR dynamics around its central black hole and the characterization of the outflows associated to emission features from Si iv to molecular lines.

Finally, we note that the estimates of $\mu$ and $\lambda_{\text {Edd }}$ presented in this work are based on the comparison of the SE measurements of $M_{\mathrm{BH}}$ with the direct SMBH mass measurement by Saturni et al. (2016), which relies, in turn, on the reverberation mapping of the high-ionization $\mathrm{C}$ IV and $\mathrm{Si}$ IV lines that are affected by narrow (intrinsic or intervening) and broad absorption. Such absorption may bias the determination of $M_{\mathrm{BH}}$, and this bias is difficult to quantify. Future observations aimed at directly measuring APM 08279+5255 $M_{\mathrm{BH}}$ with novel techniques, such as BLR spectroastrometry, are therefore needed. In particular, the spectroastrometric technique presented in Stern et al. (2015) may potentially allow to spatially resolve the kinematics of broadline regions with single spectroscopic observations taken at high signal-to-noise ratio $(S / N \gtrsim 40)$ in adaptive optics $(\mathrm{AO})$ regime. Such capabilities are already at reach of current-generation telescopes such as the Large Binocular Telescope (LBT). Therefore, the AO infrared spectroscopy of APM $08279+5255$ is a primary task to place better constraints on its $\mathrm{SMBH}$ mass and thus to obtain a more robust evaluation of lens magnification and accretion properties.

Acknowledgements. We thank our anonymous referee for their helpful comments. We acknowledge R. Maiolino (Kavli Institute for Cosmology) and D. Trevese ("Sapienza" University of Rome) for useful discussion. This research is based on observations made with the Italian Telescopio Nazionale Galileo (TNG) operated on the island of La Palma by the Fundación Galileo Galilei of the INAF (Istituto Nazionale di Astrofisica) at the Spanish Observatorio del Roque de los Muchachos of the Instituto de Astrofísica de Canarias. IRAF is distributed by the National Optical Astronomy Observatories, which are operated by the Association of the Universitiesfor Research in Astronomy, Inc., under cooperative agreement with the National Science Foundation. CC and CF acknowledge funding from the European Union's Horizon 2020 Research and Innovation Programme under the Marie Sklodowska-Curie grant agreement No. 664931. GV acknowledges funding support from the DFG Cluster of Excellence "Origin and Structure of the Universe" (www . universe-cluster . de).

\section{References}

Allen, J. T., Hewett, P. C., Maddox, N., Richards, G. T., \& Belokurov, V. 2011, MNRAS, 410, 860

Barlow, T. A. 1993, PhD Thesis, California University, USA

Baskin, A., \& Laor, A. 2005, MNRAS, 356, 1029

Bischetti, M., Piconcelli, E., Vietri, G., et al. 2017, A\&A, 598, A122

Bisogni, S., Marconi, A., \& Risaliti, G. 2017, MNRAS, 464, 385

Bongiorno, A., Maiolino, R., Brusa, M., et al. 2014, MNRAS, 443, 2077

Boroson, T. A., \& Green, R. F. 1992, ApJS, 80, 109

Capellupo, D. M., Hamann, F., Shields, J. C., Rodríguez Hidalgo, P., \& Barlow, T. A. 2011, MNRAS, 413, 908

Capellupo, D. M., Hamann, F., Shields, J. C., Rodríguez Hidalgo, P., \& Barlow, T. A. 2012, MNRAS, 422, 3249

Capellupo, D. M., Hamann, F., Shields, J. C., Halpern, J. P., \& Barlow, T. A. 2013, MNRAS, 429, 1872

Chartas, G., Brandt, W. N., Gallagher, S. C., \& Garmire, G. P. 2002, ApJ, 579, 169

Cicone, C., Brusa, M., Ramos Almeida, C., et al. 2018, Nat. Astron., 2, 176 
Coatman, L., Hewett, P. C., Banerji, M., et al. 2017, MNRAS, 465, 2120

Dong, X.-B., Wang, T.-G., Wang, J.-G., et al. 2009, ApJ, 703, L1

Dorner, B., Giardino, G., Ferruit, P., et al. 2016, A\&A, 592, A113

Downes, D., Neri, R., Wiklind, T., Wilner, D. J., \& Shaver, P. A. 1999, ApJ, 513, L1

Egami, E., Neugebauer, G., Soifer, B. T., et al. 2000, ApJ, 535, 561

Ellison, S. L., Lewis, G. F., Pettini, M., et al. 1999, PASP, 111, 946

Ellison, S. L., Ibata, R., Pettini, M., et al. 2004, A\&A, 414, 79

Fabian, A. C., Celotti, A., \& Erlund, M. C. 2006, MNRAS, 373, L16

Farrah, D., Lacy, M., Priddey, R., Borys, C., \& Afonso, J. 2007, ApJ, 662, L59

Farrah, D., Urrutia, T., Lacy, M., et al. 2012, ApJ, 745, 178

Feruglio, C., Fiore, F., Carniani, S., et al. 2015, A\&A, 583, A99

Feruglio, C., Ferrara, A., Bischetti, M., et al. 2017, A\&A, 608, A30

Filiz Ak, N., Brandt, W. N., Hall, P. B., et al. 2014, ApJ, 791, 88

Gallerani, S., Maiolino, R., Juarez, Y., et al. 2010, A\&A, 523, A85

Gibson, R. R., Brandt, W. N., Schneider, D. P., \& Gallagher, S. C. 2008, ApJ, 675,985

Gibson, R. R., Jiang, L., Brandt, W. N., et al. 2009, ApJ, 692, 758

Gibson, R. R., Brandt, W. N., Gallagher, S. C., Hewett, P. C., \& Schneider, D. P. 2010, ApJ, 713, 220

Glikman, E., Helfand, D. J., \& White, R. L. 2006, ApJ, 640, 579

Graham, A. W., Onken, C. A., Athanassoula, E., \& Combes, F. 2011, MNRAS 412, 2211

Grier, C. J., Martini, P., Watson, L. C., et al. 2013, ApJ, 773, 90

Hagino, K., Done, C., Odaka, H., Watanabe, S., \& Takahashi, T. 2017, MNRAS, 468,1442

Hasinger, G., Schartel, N., \& Komossa, S. 2002, ApJ, 573, L77

Hewett, P. C., \& Foltz, C. B. 2003, AJ, 125, 1784

Hines, D. C., Schmidt, G. D., \& Smith, P. S. 1999, ApJ, 514, L91

Ibata, R. A., Lewis, G. F., Irwin, M. J., Lehár, J., \& Totten, E. J. 1999, AJ, 118, 1922

Irwin, M. J., Ibata, R. A., Lewis, G. F., \& Totten, E. J. 1998, ApJ, 505, 529

Kaspi, S., Brandt, W. N., Maoz, D., et al. 2007, ApJ, 659, 997

Knigge, C., Scaringi, S., Goad, M. R., \& Cottis, C. E. 2008, MNRAS, 386, 1426

Labiano, A., Azzollini, R., Bailey, J., et al. 2016, in Observatory Operations: Strategies, Processes, and Systems VI, Proc. SPIE, 9910, 99102W

Ledoux, C., Theodore, B., Petitjean, P., et al. 1998, A\&A, 339, L77

Lewis, G. F., Robb, R. M., \& Ibata, R. A. 1999, PASP, 111, 1503

Lewis, G. F., Carilli, C., Papadopoulos, P., \& Ivison, R. J. 2002a, MNRAS, 330, L15

Lewis, G. F., Ibata, R. A., Ellison, S. L., et al. 2002b, MNRAS, 334, L7

Lundgren, B. F., Wilhite, B. C., Brunner, R. J., et al. 2007, ApJ, 656, 73

Marconi, A., Axon, D. J., Maiolino, R., et al. 2008, ApJ, 678, 693

Marconi, A., Axon, D. J., Maiolino, R., et al. 2009, ApJ, 698, L103

Markwardt, C. B. 2009, in Astronomical Data Analysis Software and Systems XVIII, eds. D. A. Bohlender, D. Durand, \& P. Dowler, ASP Conf. Ser., 411, 251

Marziani, P., Sulentic, J. W., Plauchu-Frayn, I., \& del Olmo A. 2013, ApJ, 764, 150

Marziani, P., Martínez Carballo, M. A., Sulentic, J. W., et al. 2016, Ap\&SS, 361, 29
Netzer, H., Shemmer, O., Maiolino, R., et al. 2004, ApJ, 614, 558

Ojha, R., Zacharias, N., Hennessy, G. S., Gaume, R. A., \& Johnston, K. J. 2009, AJ, 138,845

Onken, C. A., Ferrarese, L., Merritt, D., et al. 2004, ApJ, 615, 645

Oyabu, S., Kawara, K., Tsuzuki, Y., et al. 2009, ApJ, 697, 452

Pancoast, A., Brewer, B. J., Treu, T., et al. 2014, MNRAS, 445, 3073

Pancoast, A., Brewer, B. J., Treu, T., et al. 2015, MNRAS, 448, 3070

Park, D., Kelly, B. C., Woo, J.-H., \& Treu, T. 2012, ApJS, 203, 6

Pei, Y. C. 1992, ApJ, 395, 130

Peterson, B. M. 1997, An Introduction to Active Galactic Nuclei (Cambridge, New York: Cambridge University Press)

Petitjean, P., Aracil, B., Srianand, R., \& Ibata, R. 2000, A\&A, 359, 457

Proga, D., \& Kallman, T. R. 2004, ApJ, 616, 688

Reichard, T. A., Richards, G. T., Hall, P. B., et al. 2003, AJ, 126, 2594

Richards, G. T., Vanden Berk, D. E., Reichard, T. A., et al. 2002, AJ, 124, 1

Richards, G. T., Hall, P. B., Vanden Berk, D. E., et al. 2003, AJ, 126, 1131

Richards, G. T., Kruczek, N. E., Gallagher, S. C., et al. 2011, AJ, 141, 167

Riechers, D. A., Walter, F., Carilli, C. R., \& Lewis, G. F. 2009, ApJ, 690, 463

Risaliti, G., \& Elvis, M. 2010, A\&A, 516, A89

Rowan-Robinson, M. 2000, MNRAS, 316, 885

Runnoe, J. C., Brotherton, M. S., \& Shang, Z. 2012a, MNRAS, 427, 1800

Runnoe, J. C., Brotherton, M. S., \& Shang, Z. 2012b, MNRAS, 422, 478

Saez, C., \& Chartas, G. 2011, ApJ, 737, 91

Saez, C., Chartas, G., \& Brandt, W. N. 2009, ApJ, 697, 194

Saturni, F. G., Trevese, D., Vagnetti, F., \& Perna, M. 2014, Adv. Space Res., 54 1434

Saturni, F. G., Trevese, D., Vagnetti, F., Perna, M., \& Dadina, M. 2016, A\&A, 587, A43

Shen, Y. 2016, ApJ, 817, 55

Shen, Y., \& Ho, L. C. 2014, Nature, 513, 210

Shen, Y., \& Liu, X. 2012, ApJ, 753, 125

Shen, Y., Richards, G. T., Strauss, M. A., et al. 2011, ApJS, 194, 45

Srianand, R., \& Petitjean, P. 2000, A\&A, 357, 414

Stern, J., Hennawi, J. F., \& Pott, J.-U. 2015, ApJ, 804, 57

Trevese, D., Paris, D., Stirpe, G. M., Vagnetti, F., \& Zitelli, V. 2007, A\&A, 470, 491

Trevese, D., Saturni, F. G., Vagnetti, F., et al. 2013, A\&A, 557, A91

Trevese, D., Perna, M., Vagnetti, F., Saturni, F. G., \& Dadina, M. 2014, ApJ, 795, 164

Trump, J. R., Hall, P. B., Reichard, T. A., et al. 2006, ApJS, 165, 1

Tsuzuki, Y., Kawara, K., Yoshii, Y., et al. 2006, ApJ, 650, 57

Urrutia, T., Becker, R. H., White, R. L., et al. 2009, ApJ, 698, 1095

Vanden Berk, D. E., Richards, G. T., Bauer, A., et al. 2001, AJ, 122, 549

Véron-Cetty, M.-P., Joly, M., \& Véron, P. 2004, A\&A, 417, 515

Vestergaard, M., \& Peterson, B. M. 2006, ApJ, 641, 689

Vestergaard, M., \& Wilkes, B. J. 2001, ApJS, 134,

Vietri, G., Piconcelli, E., Bischetti, M., et al. 2018, A\&A, 617, A81

Weymann, R. J., Morris, S. L., Foltz, C. B., \& Hewett, P. C. 1991, ApJ, 373, 23

Wu, J., Vanden Berk, D. E., Brandt, W. N., et al. 2009, ApJ, 702, 767

Zuo, W., Wu, X.-B., Fan, X., et al. 2015, ApJ, 799, 189 